Abstract A planar Babinet-inverted dimer metamaterial possessing strong optical activity is proposed and characterized. An original fabrication method to produce large area (up to several $\mathrm{cm}^{2}$ ) freely suspended flexible metallic membranes is implemented to fabricate the metamaterial. Its optical properties are characterized by terahertz time-domain spectroscopy, revealing anisotropic transmission with high optical activity. A simple coupled resonator model is applied to explain the principal optical features of the dimers, with predictive power of positions and number of resonances through a parametrical model. The model is validated for correct polarization-dependent quantitative results on the optical activity in transmission spectra. The fabrication method presented in this work as well as the slit dimer design has great potential for exploitation in terahertz
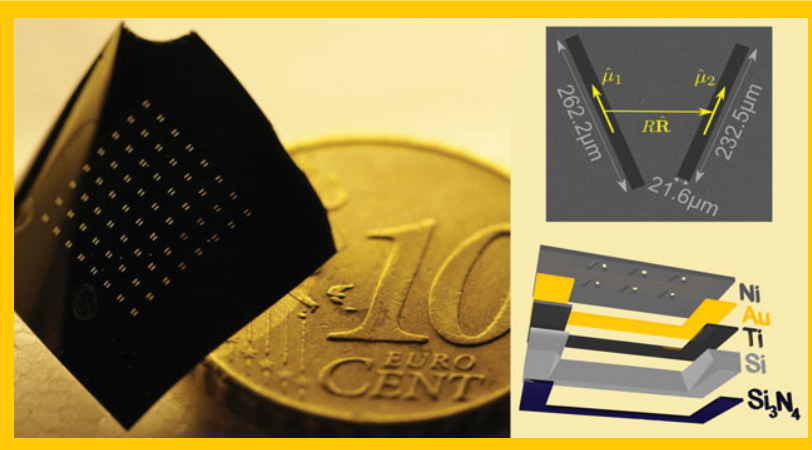
optics.

\title{
Optically active Babinet planar metamaterial film for terahertz polarization manipulation
}

\author{
Maksim Zalkovskij ${ }^{1, *}$, Radu Malureanu ${ }^{1}$, Christian Kremers ${ }^{2}$, Dmitry N. Chigrin ${ }^{2,3}$, \\ Andey Novitsky ${ }^{1,4}$, Sergei Zhukovsky ${ }^{1}$, Peter T. Tang ${ }^{5}$, Peter U. Jepsen ${ }^{1}$, \\ and Andrei V. Lavrinenko ${ }^{1}$
}

\section{Introduction}

Terahertz $(\mathrm{THz})$ radiation is widely employed in a broad range of fields, e.g. in biology, medicine, communication, security, chemistry, and spectroscopy [1-3]. To expand the application of terahertz radiation in the aforementioned fields new device designs and fabrication methods are needed. The ability of metamaterials to manipulate the electromagnetic waves makes them natural candidates for THz optical components [4].

Various chiral metamaterial designs have already been proposed and characterized [5-12]. Many of the proposed designs are complex (e.g. consisting of multi-layer or truly three dimensional structures) making them hard to fabricate or integrate into a THz-optic component. On the other hand, the majority of metamaterials for the $\mathrm{THz}$ region are supported by either a bulk silicon wafer or thin layer of material such as polyimide, silicon nitride, silk or paper, which inevitably leads to increased optical losses [13-16]. In this paper, we propose a novel fabrication method for large area, freestanding, micrometer thick flexible planar metamaterials (PMMs).

Within a simple geometric framework, PMMs can acquire pronounced optical activity relative to their thickness.
We follow the concept of asymmetric nano-rod dimers with straight, non-parallel elements of different sizes, as theoretically described in [17]. This structure mimics the widely used asymmetric split ring geometry [6], though with straightened segments. It was shown that this structure possesses strong optical activity due to elliptical dichroism [17]. In our case, instead of having a metamaterial consisting of metallic rods, we chose its inverse (Babinet) counterpart, where the rods are substituted by hollow elements (antirods) in a metal membrane [18]. According to the Babinet principle $[19,20]$, spectral properties of such mutually inverse structures (rods and slots) are predicted to be similar, but inverted. To differentiate our PMM from that reported in [17], we refer to our design as the Babinet asymmetric dimer. The Babinet structure makes it possible to design and fabricate a freestanding PMM film consisting only of metal. Besides the advantages with respect to fabrication and mechanical properties, the Babinet design resonantly increases transmission near the frequencies where the PMM displays the highest optical activity.

We have developed a fabrication process to grow at least $2 \mu \mathrm{m}$ thick nickel (Ni) PMM membranes with areas from $8 \times 8 \mathrm{~mm}^{2}$. The thickness of the Ni metamaterial film is limited primarily by the thickness of the photoresist (PR)

\footnotetext{
${ }^{1}$ Department of Photonics Engineering, Technical University of Denmark, DK-2800 Kongens Lyngby, Denmark

${ }^{2}$ Institute of High-Frequency and Communication Technology, University of Wuppertal, 42119 Wuppertal, Germany

${ }^{3}$ Current address: I. Physikalisches Institut, RWTH Aachen, D-52056 Aachen, Germany

${ }^{4}$ Department of Theoretical Physics and Astrophysics, Belarusian State University, 220030 Minsk, Belarus

${ }^{5}$ IPU, Technology Development, Produktionstorvet 425, DK-2800 Kongens Lyngby, Denmark

*Corresponding author: e-mail: mzal@fotonik.dtu.dk
} 
(a)

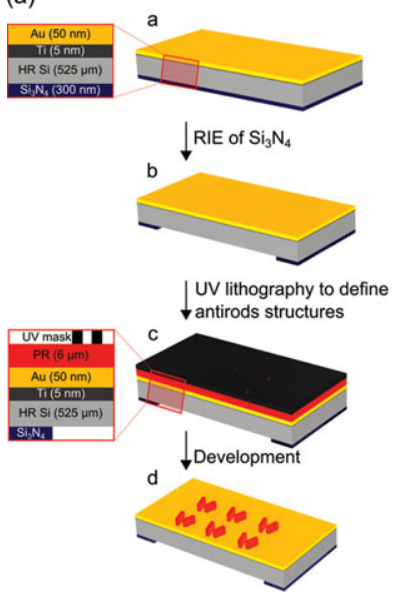

(b)

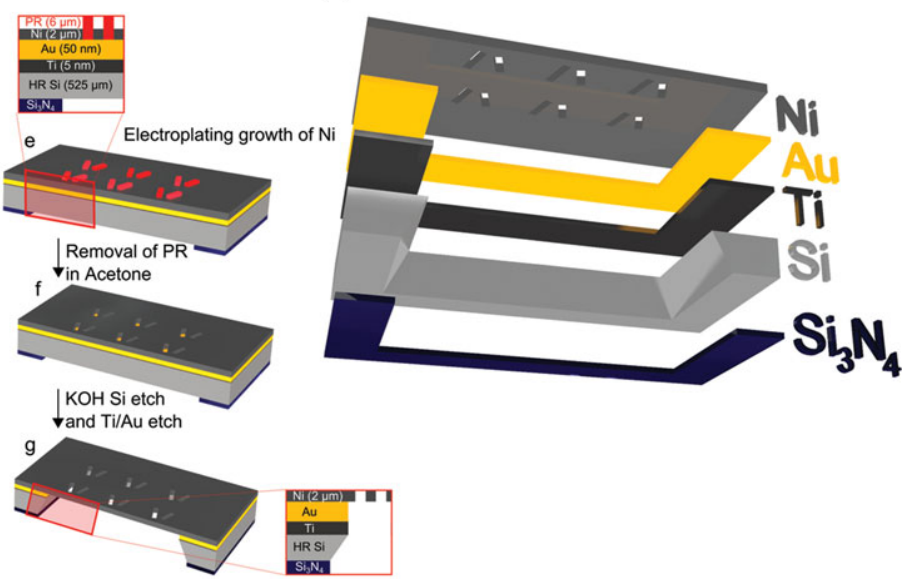

Figure 1 (a) Main fabrication steps and (b) schematic overview of the structure. Not drawn to scale.

used to define the surface structures. The dimensions which can be reached with our process are difficult to achieve by traditional lift-off processes due to the following factors. The deposition of metals on the photoresist sidewalls during the evaporation process is critical to avoid for the lift-off. This is because the overall shape of the metallic structures depends on the amount of metal deposited on the sidewalls, and this cannot be accurately controlled with such a thick PR. Additionally, one needs a rather high ratio, on the order of at least 5:1, between the thickness of the PR itself and the metal deposited on top of it, and it is difficult to perform precise UV-lithography on a very thick PR with sufficient final resolution of the pattern. Our process clears away these limitations.

In Sections 2 and 3 we describe the fabrication process and confirm the quality of the fabricated films by optical characterization. In Section 4 we discuss a theoretical model that describes the behavior of our freely suspended PMM design. In Section 5 we discuss the anisotropic properties of our samples.

\section{Fabrication}

The membranes were fabricated with an area of $8 \times 8 \mathrm{~mm}^{2}$, and a thickness of $2 \mu \mathrm{m}$. Such a huge aspect ratio (1:4000) poses various challenges in the fabrication process. The thickness of $2 \mu \mathrm{m}$ was chosen for mechanical robustness of the sample. The fabrication process leads to free-standing membranes, thus eliminating substrate influences such as increased reflection losses and Fabry-Perot interference effects. The processing steps are illustrated in Fig. 1(a), with the final layer structure shown in Fig. 1(b). The structure of the fabricated metamaterial is shown in Fig. 2(a) and is defined by the following target dimensions: the lengths $l_{1}=260 \mu \mathrm{m}$ and $l_{2}=230 \mu \mathrm{m}$, width $d=20 \mu \mathrm{m}$, spacing $\Delta=160 \mu \mathrm{m}$ of the two slots, angle $\alpha=45^{\circ}$ between them, and periodicities $\Lambda_{\mathrm{x}}=\Lambda_{\mathrm{y}}=500 \mu \mathrm{m}$.
In the fabrication flow, we start with a double-side polished $525 \mu \mathrm{m}$-thick Si wafer. On the front side we evaporate a double layer of $\mathrm{Ti} / \mathrm{Au}$. The $5 \mathrm{~nm}$ thin Ti layer is used to promote the adhesion of gold to the substrate, while the $50 \mathrm{~nm}$ of $\mathrm{Au}$ is the seed layer for the electrochemical growth of the $\mathrm{Ni}$ membrane. On the backside of the substrate, $300 \mathrm{~nm}$ of $\mathrm{Si}_{3} \mathrm{~N}_{4}$ is deposited by plasma-enhanced chemical vapor deposition (Fig. 1(a.a)). The bottom layer of $\mathrm{Si}_{3} \mathrm{~N}_{4}$ is needed to protect the edges of the Si wafer from etching with $\mathrm{KOH}$, depicted in the step between (Fig 1(a.f)) and (Fig 1(a.g)). After these initial steps (Fig. 1(a.a)), the membrane aperture is defined through standard UV-lithography exposure on the backside of the substrate. The next step is the removal of the $\mathrm{Si}_{3} \mathrm{~N}_{4}$ by reactive ion etching using the PR as a mask. The result can be seen schematically in (Fig. 1(a.b)). Once the membrane aperture position is defined, the rest of the process steps are performed relative to it. We then spin a thick (approximately $6 \mu \mathrm{m}$ ) PR on the top side of the wafer and, using aligned exposure with the substrate backside, define the structures on top of the $\mathrm{Si}_{3} \mathrm{~N}_{4}$ aperture (Fig. 1(a.c)). After the development of the PR (Fig. 1(a.d)) we grow $2 \mu \mathrm{m}$ of Ni using electroplating (Fig. 1(a.e)). The nickel electroplating electrolyte is of the sulphamate type, and is optimized for low internal stress, small grain size, and zero defects. The electrolyte is operated at a relatively low temperature of $32{ }^{\circ} \mathrm{C}$, using mild agitation with purified compressed air and continuous monitoring of internal stress and $\mathrm{pH}$ value. The nickel is deposited at a current density of $1.38 \mathrm{~A} / \mathrm{dm}^{2}$ corresponding to a deposition rate of $0.25 \mu \mathrm{m} / \mathrm{min}$. The next step is the removal of the remaining $\mathrm{PR}$ in acetone, resulting in the desired Ni structure on the $\mathrm{Si} / \mathrm{Au}$ substrate (Fig. 1(a.f)). The $\mathrm{Si}$ wafer is then back-etched in an $80{ }^{\circ} \mathrm{C} \mathrm{KOH}$ bath. Here, the $\mathrm{Si}_{3} \mathrm{~N}_{4}$ layer acts as etch mask. The etching rate is approximately $1.3 \mu \mathrm{m} / \mathrm{min}$, and therefore this is the longest step in the fabrication process, with duration of approximately 6-7 hours. The $\mathrm{KOH}$ etch is easily controllable and is not aggressive to the $\mathrm{Ni}$ membrane ( $\mathrm{Ni}$ is passive in most high $\mathrm{pH}$ solutions), so the structure definition is preserved 

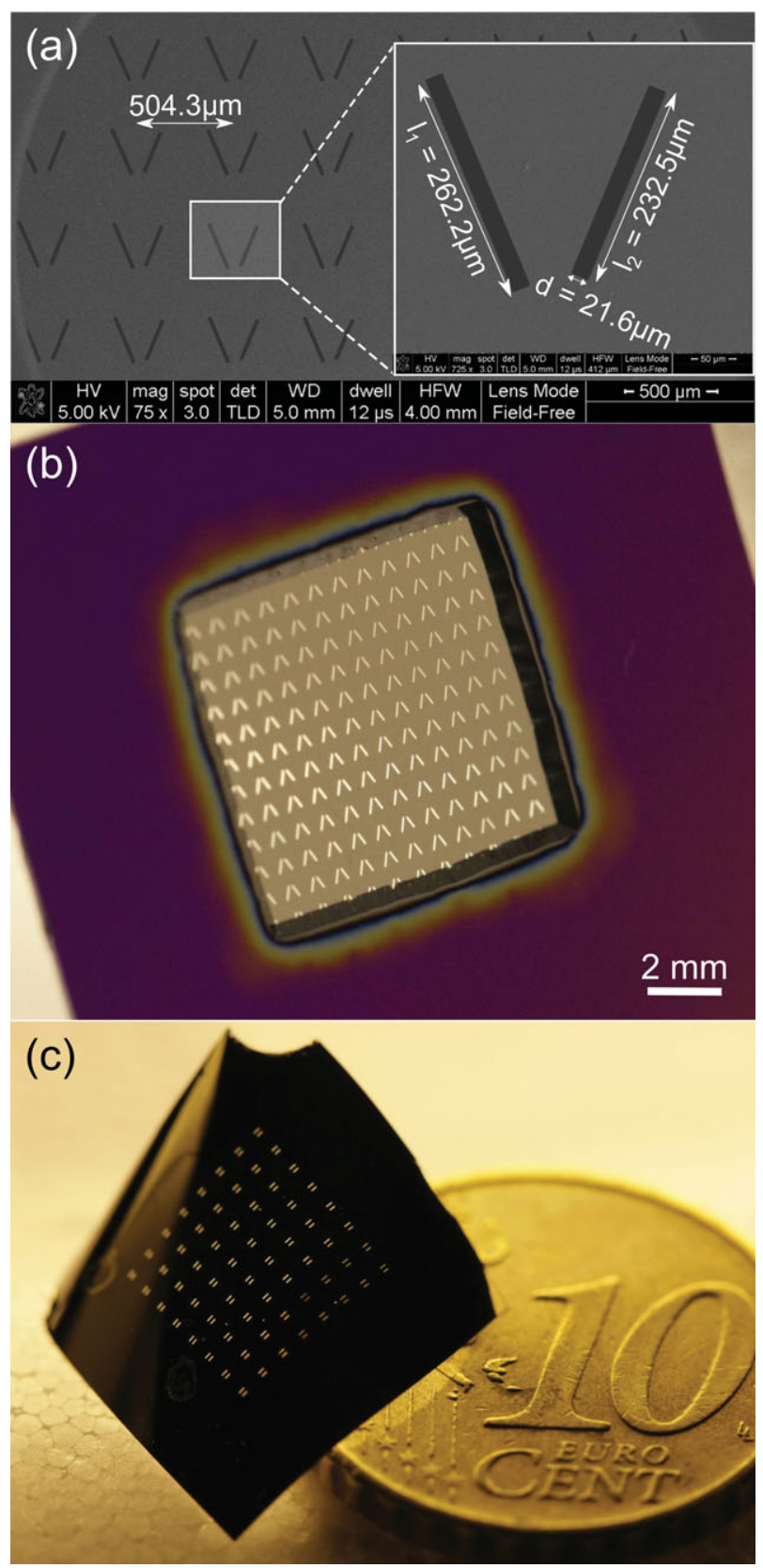

Figure 2 (a) SEM image of V-shape sample with a period of $504.3 \mu \mathrm{m}$. The inset displays a single unit cell of the sample. (b) Back side of the sample. Silicon is used as the frame to handle the sample during the measurements. The back side of the membrane is visible. (c) Membrane detached from the Si frame. The membrane, although very thin, is resistant enough to be removed from the Si frame and used as flexible $\mathrm{THz}$ PMM. The 10 cent coin is used for size comparison.

throughout the etch process. The last step in the process is the selective removal of the Ti/Au layer using a commercial etching solution, Entreat 100, from Engelhard, NJ, USA (Fig. 1(a.g)). A schematic overview of the completed structure can be seen in Fig. 1(b).
A scanning electron microscopy (SEM) picture of the fabricated structure is shown in Fig. 2(a), demonstrating that the features are sharply defined, with all critical design dimensions preserved. In Fig. 2(b) an optical image of the structure is shown. The Si frame can be clearly seen and, at the same time, light passes through the dimer structures, showing that all other layers behind the membrane are removed.

It should be mentioned that during the $\mathrm{KOH}$ etch of $\mathrm{Si}$, the $<111>$ crystallographic plane is inert, thus allowing for a very smooth and clean side of the frame. The membrane is easily peeled off the substrate. Figure 2(c) shows the membrane completely detached from the frame. Apart from a slight defect in the top corner, the membrane is complete and the structures are intact. This demonstrates that the $\mathrm{Ni}$ layer is sufficiently mechanically robust to serve as a flexible structure and that membranes with larger dimensions can be fabricated.

\section{Measurements}

Transmission spectra of linearly polarized light through the Babinet PMM are measured by a commercial terahertz time-domain spectroscopy system (Picometrix T-Ray 4000). The system generates and records the $\mathrm{THz}$ transients using photoconductive switches [3] that are fiber coupled to a femtosecond laser. The system operates at a scan rate of $100 \mathrm{~Hz}$, and we record the average of 100.000 waveforms (time window $320 \mathrm{ps}$, total acquisition time $17 \mathrm{~min}$ ) with spectroscopic information from 0.1 to $2.0 \mathrm{THz}$ and an electric field dynamic range of 3200 at $0.5 \mathrm{THz}(70 \mathrm{~dB}$ power dynamic range). Figure 3 displays the transmission spectra for the two orthogonal linear polarizations for both the $\mathrm{V}$-shape and parallel slots dimer PMM. Linear polarization transmission coefficients are recovered by cross polarization measurements, where the transmission through air is used as a reference, $\tau_{i j}(\omega)=E_{i j}^{\text {sample }} / E_{i i}^{\text {reference }}$, indices $i$ and $j$ can be either $x$ or $y$. The incident polarization is controlled by rotation of the samples.

At $0^{\circ}$ incident polarization (see inset of Fig. 3) two transmission resonances at 0.48 and $0.56 \mathrm{THz}$ are observed for both samples. Even though the hollow elements cover only $4.3 \%$ of the total Ni membrane area, an enhanced relative field transmission amplitude [21] as high as 0.67 is observed for the resonance at $0.56 \mathrm{THz}$. The two transmission resonances occur due to a favorable coupling between the incident field and the eigenmodes of metamaterial structure. Since the dimensions of the slot are identical for both parallel and V-shape samples, the transmission peaks appear at the same frequencies, with similar strengths and widths. The relative strength of the two resonances (which can be individually suppressed or enhanced) is influenced by the distance between the hollow asymmetric elements [22]. A parallel slot dimer PMM is geometrically similar to a wire grid polarizer, so expectedly, the transmission coefficient drops to zero when the sample is rotated by $90^{\circ}$. In contrast to this, we observe a single transmission peak at 0.5 $\mathrm{THz}$ for the V-shape sample rotated by $90^{\circ}$ (Fig. 3). As 


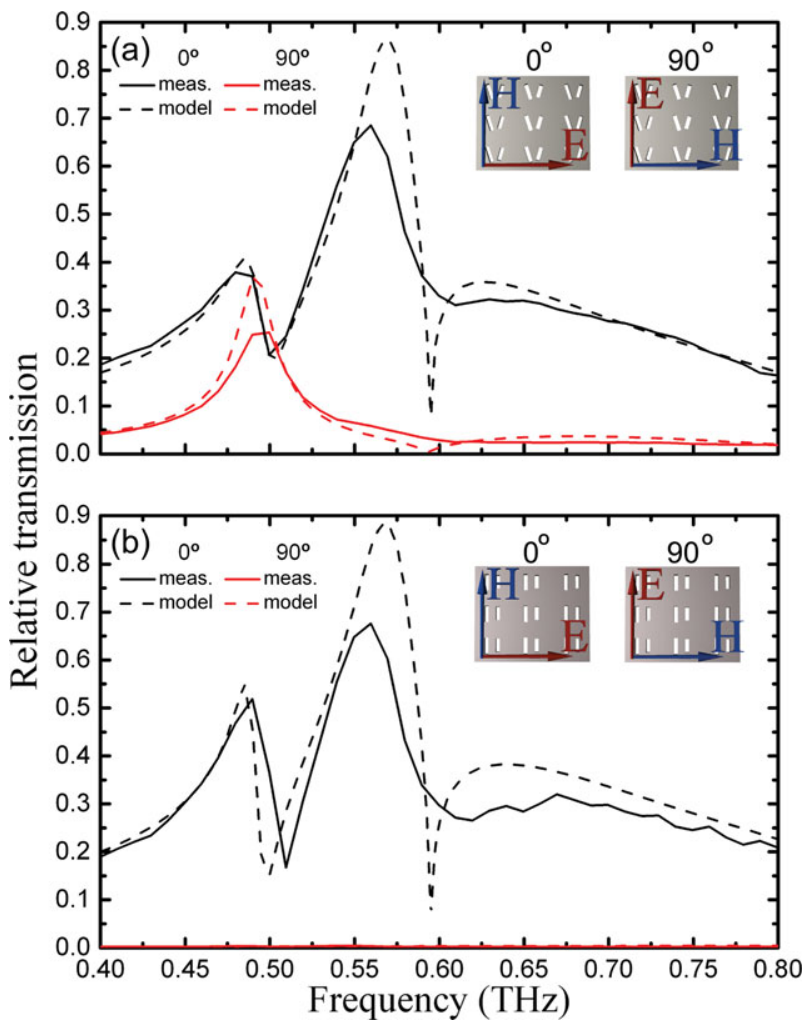

Figure 3 Relative amplitude transmission $|\tau|$ of (a) V-shaped and (b) parallel antirod planar metamaterial. Measurements (solid curves) and numerical simulation results (dashed curves) are shown for two different rotation positions of the sample, $0^{\circ}\left(\left|\tau_{x x}\right|\right.$, black curves) and $90^{\circ}\left(\left|\tau_{y y}\right|\right.$, red curves). The insets illustrate the sample orientation with respect to the incident electromagnetic fields.

will be shown below, this is the result of interplay between the coupling of the incident radiation to the PMM eigenmodes, and the corresponding transmission efficiencies of the eigenmodes themselves.

\section{Theoretical model}

The measurement results were compared with the result of full-wave numerical simulations performed using AnsysHFSS, a commercial finite element electromagnetic field solver. The dielectric function of $\mathrm{Ni}$ was modeled using a 5-pole Drude-Lorentz model [23].

The power transmission $T$ can be determined from numerical simulations as

$$
T=\frac{\int \vec{S}_{t o t} \cdot \hat{z} d A}{\int \vec{S}_{i n c} \cdot \hat{z} d A}=\mu_{0} c_{0} \frac{\int \operatorname{Re}\left[\vec{E} \times \vec{H}^{*}\right] \cdot \hat{z} d A}{A E_{i n c}^{2}},
$$

using the total fields $(\vec{E}, \vec{H})$ on the detector surface $A$ below and parallel to the Ni membrane. Here $\mu_{0}, c_{0}$ and $E_{\text {inc }}$ denote the vacuum permeability, vacuum speed of light, and electric field amplitude of the incident plane wave, respectively.
The amplitude linear-polarization transmission coefficients $\tau_{i j}$ for an $\hat{x}_{j}$-polarized incident wave are calculated as

$$
\tau_{i j}=\frac{\int \vec{E} \cdot \hat{x}_{i} d A}{\int \vec{E}_{i n c} \cdot \hat{x}_{j} d A}
$$

Figure 3 shows good quantitative agreement between the measurements and modeling results. The sharp dip seen at $0.6 \mathrm{THz}$ in the simulated spectra results from lattice effects (the Rayleigh anomaly), when the wavelength of light equals the lattice period. In experiment, the PMM interacts with a finite-sized beam rather than an infinitely extended plane wave, therefore, this lattice-related dip is much less pronounced (but still visible) in the measured spectra. For the same reason, the second resonance (at $0.56 \mathrm{THz}$ ) is more pronounced in simulations than in measurements, and is slightly shifted in frequency by $10 \mathrm{GHz}$. Some minor deviations may also be attributed to small differences between the model dielectric function of $\mathrm{Ni}$ and that of the actual, grown layer.

To obtain a deeper physical understanding of spectral properties of the Babinet PMM we employ a coupleddipole theory developed previously for plasmonic nanorod dimer metamaterials [17,24]. Making note of the fact that a nanoslot antenna can be described by a bound-charge oscillator model similar to a nanorod antenna [25] by virtue of the Babinet principle, we can assume that each individual slot responds to the incident harmonically oscillating electric field $\mathbf{E}_{0}$ with an induced dipole moment $\mathbf{d}_{1,2}=\boldsymbol{\alpha}_{1,2} \mathbf{E}_{0}$, where the polarizabilities $\boldsymbol{\alpha}_{1,2}$ are tensors of the form

$$
\begin{aligned}
\alpha_{j=1,2} & =\frac{f_{j}}{\omega_{j}^{2}-\omega^{2}-i \omega\left(\gamma_{j}+\delta_{j} \omega^{2}\right)} \hat{\mu}_{j} \otimes \hat{\mu}_{j} \\
& =\alpha_{j}(\omega) \hat{\mu}_{j} \otimes \hat{\mu}_{j} .
\end{aligned}
$$

Here the expression $\hat{\mu}_{j} \otimes \hat{\mu}_{j}$ denotes the outer (dyadic) product of the unit vector pointing along the slot orientation with itself (see Fig. 4). The parameters $f_{j}, \omega_{j}, \gamma_{j}$, and $\delta_{j}$ are determined by fitting the results of numerical simulations.

When two slots are coupled together in a dimer, their induced dipole moments are modified by the presence of each other. This can be accounted for by the Green function approach [24], resulting in a system of coupled equations for $\mathbf{d}_{1,2}$ and the following form of the total effective dipole polarizability of the dimer

$$
\begin{aligned}
\alpha^{\mathrm{eff}}= & \frac{\alpha_{1} \hat{\mu}_{1} \otimes \hat{\mu}_{1}}{1-\kappa^{2} \alpha_{1} \alpha_{2}}+\frac{\alpha_{2} \hat{\mu}_{2} \otimes \hat{\mu}_{2}}{1-\kappa^{2} \alpha_{1} \alpha_{2}}+\frac{\kappa \alpha_{1} \alpha_{2}}{1-\kappa^{2} \alpha_{1} \alpha_{2}} \\
& \times\left(\hat{\mu}_{1} \otimes \hat{\mu}_{2}+\hat{\mu}_{2} \otimes \hat{\mu}_{1}\right) .
\end{aligned}
$$

where $\kappa$ is the coupling coefficient determined by the relative arrangement of the slot elements. The detailed procedure of its calculation can be found elsewhere [17]. Neglecting interaction between the neighboring dimers in the lattice, we can determine the effective permittivity tensor of the metamaterial as $\varepsilon^{\text {eff }}=1-\left(\varepsilon_{0} V_{\text {cell }}\right)^{-1} \alpha^{\text {eff }}$. 


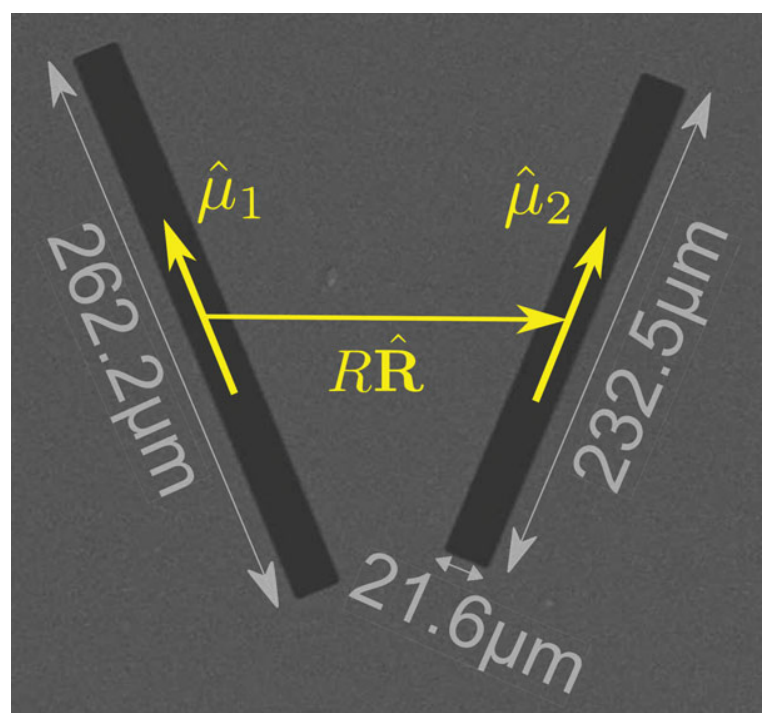

Figure 4 SEM image of unit cell showing the unit vectors defining the dimer geometry.

Representing the actual metamaterial as a slab of this effective material, we can then use the generalized transfermatrix approach $[26,27]$ to determine the reflection and transmission spectra. Previous accounts demonstrate a good agreement of this approach with direct numerical simulations for rod dimers [17,24].

Figure 5 compares the analytically calculated transmission spectra for the $\mathrm{V}$-shaped and parallel dimer structures with the results of the full-wave simulation. We see that by properly choosing the parameters in Eq. (3), a very good qualitative agreement can be achieved, thus confirming that the coupled dipole model captures the underlying physics of wave interaction with the slot dimer metamaterials.

We can now explain why there are two peaks in $\tau_{x x}$ and only one in $\tau_{y y}$, for the V-shaped structures. From the analytical description given above, we can determine the polarization eigenstates [26] $\mathbf{e}_{1,2}$ of the metamaterial, i.e., polarization states that remain unchanged as a wave passes through the metamaterial. An arbitrarily polarized incident wave can be now obtained by its expantion in the eigenstates basis,

$$
\mathbf{E}_{\alpha}(\omega)=C_{\alpha}^{1}(\omega) \mathbf{e}_{1}(\omega)+C_{\alpha}^{2}(\omega) \mathbf{e}_{2}(\omega), \quad \alpha=x, y,
$$

leading to the following form of the transmission components $\tau_{\alpha \beta}$ of Eq. (2),

$$
\tau_{\alpha \beta}=C_{\beta}^{1}(\omega) t_{1}(\omega) C_{\alpha}^{1}(\omega)+C_{\beta}^{2}(\omega) t_{2}(\omega) C_{\alpha}^{2}(\omega),
$$

where $t_{1,2}(\omega)$ are transmission spectra of the eigenmodes. This finally results in

$$
\begin{aligned}
\tau_{x x} & =\left[C_{x}^{1}(\omega)\right]^{2} t_{1}(\omega)+\left[C_{x}^{2}(\omega)\right]^{2} t_{2}(\omega), \\
\tau_{y y} & =\left[C_{y}^{1}(\omega)\right]^{2} t_{1}(\omega)+\left[C_{y}^{2}(\omega)\right]^{2} t_{2}(\omega) .
\end{aligned}
$$
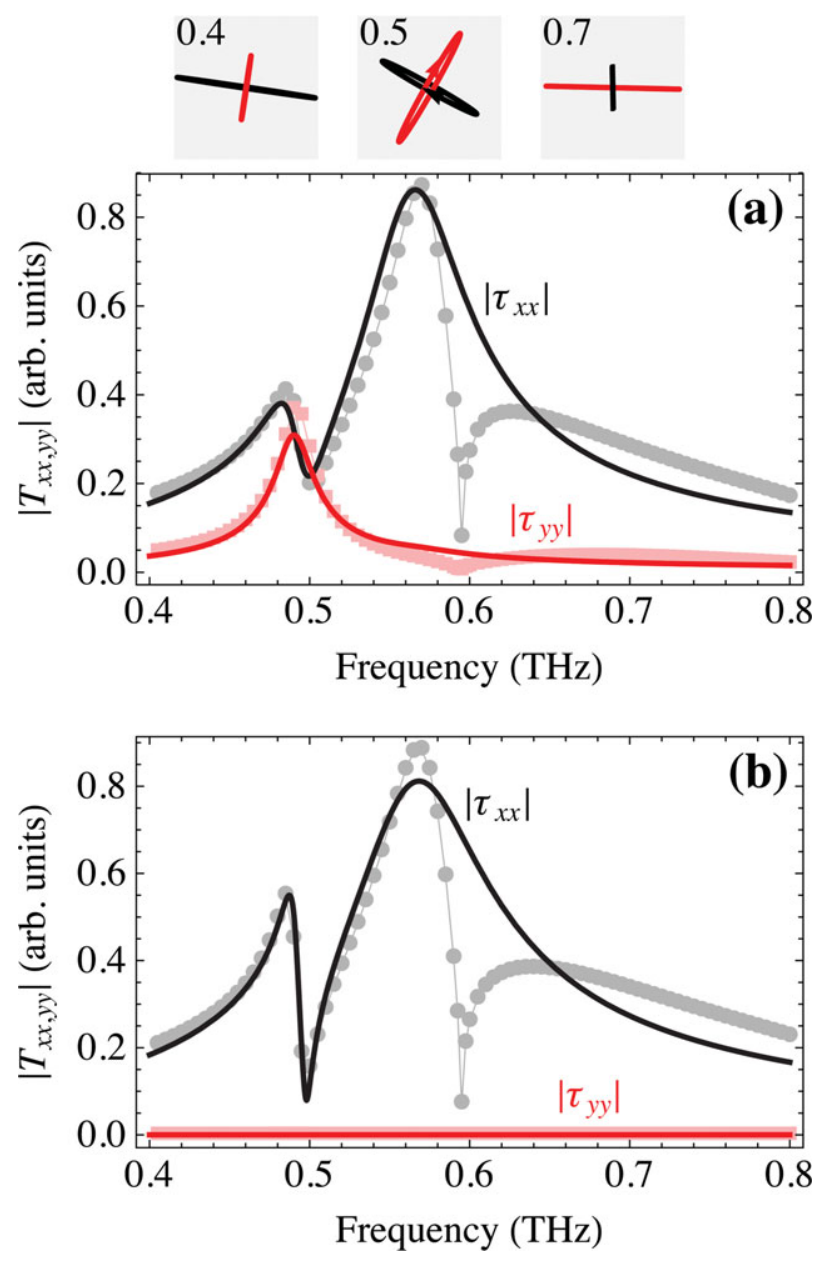

Figure 5 Transmission spectra $\tau_{x x}$ and $\tau_{y y}$ for (a) V-shaped and (b) parallel-shaped dimer metamaterials, analytically determined from the proposed theory (lines) compared to direct numerical simulations (dots). The dimer dimensions are shown in Fig. 2a. The insets show the eigenpolarizations for three different frequencies $(0.4,0.5$, and $0.7 \mathrm{THz})$.

As seen in Fig. 6, the transmission spectrum for the two eigenmodes $t_{1}(\omega)$ and $t_{2}(\omega)$ have a resonance peak at their respective frequencies, associated with the presence of two rods with different length (see Fig. 4). However, the expansion coefficients $C^{1,2}(\omega)$ are flipped near a certain frequency between these resonance peaks, so a linearly polarized incident wave predominantly couples to the mode $\mathbf{e}_{1}$ at lower frequencies, and to the mode $\mathbf{e}_{2}$ for higher frequencies. This is a purely geometrical effect, resulting from a specific dependence of polarization eigenstates on frequency, $\mathbf{e}_{1,2}(\omega)$, as seen in the insets in Fig. 5, and determined by the mutual orientation of the slots in the dimer.

When the incident wave polarization is rotated, the coupling coefficients in Eq. (5) are expectedly reversed, $C_{x}^{1}(\omega)=C_{y}^{2}(\omega), C_{y}^{1}(\omega)=C_{x}^{2}(\omega)$. Hence, for one polarization the peaks in $t_{1,2}(\omega)$ coincide in frequency with regions where $C^{1,2}(\omega)$ is large. As a result, Eq. (6) gives a twopeak dependence that corresponds with the two eigenmode 

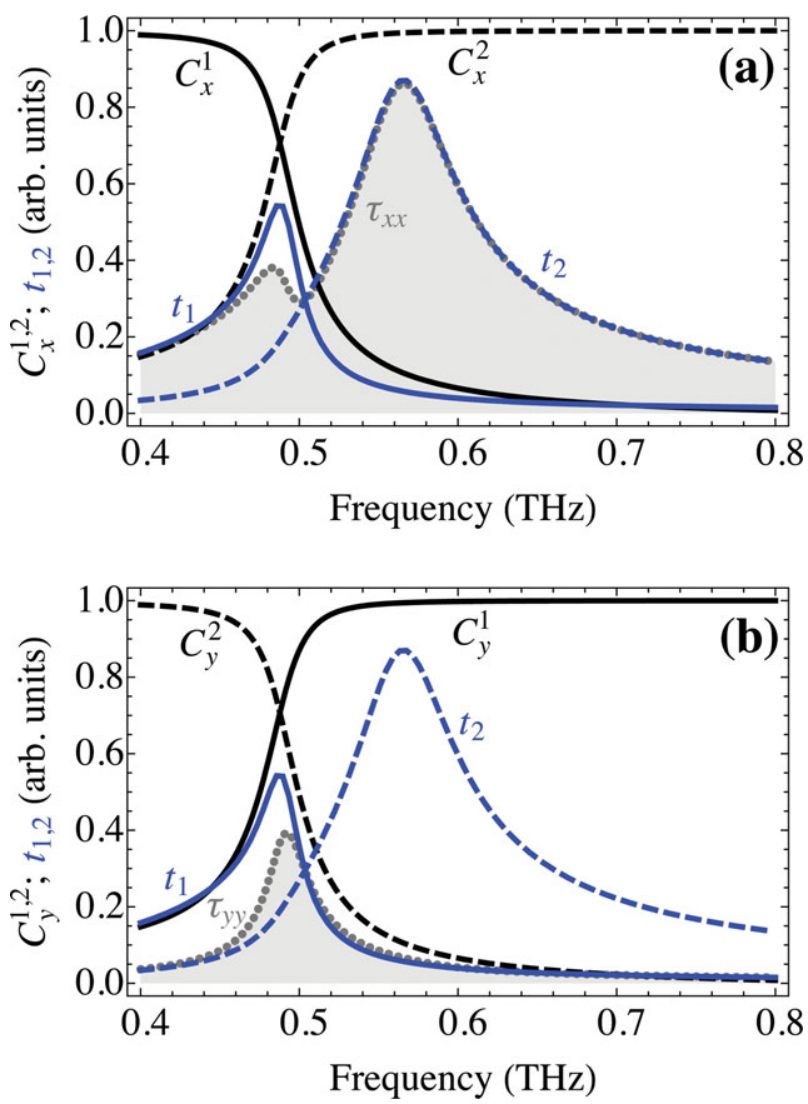

Figure 6 Eigenmode transmission coefficients $t_{1,2}(\omega)$ together with the eigenmode expansion coefficients for the incident light linearly polarized (a) along the x-axis: $C_{X}{ }^{1,2}(\omega)$; (b) along the $y$ axis: $C_{y}{ }^{1,2}(\omega)$. The dotted lines with shading denote the resulting transmission $\tau_{x x}$ and $\tau_{y y}$ given by Eq. (6).

resonances. For the other polarization, strong $t_{1,2}(\omega)$ at the peaks is cancelled by weak $C^{1,2}(\omega)$ at those frequencies, so a significant response is only seen where the four quantities, $t_{1,2}(\omega)$ and $C^{1,2}(\omega)$, are all significantly non-zero. This is the case only in the overlap region between the eigenstate resonances, where the eigenpolarizations display sufficient ellipticity, resulting in a weaker response with only one peak.

In contrast to the $\mathrm{V}$-shaped dimers, the parallel dimers can only have linearly polarized eigenmodes, which is seen from Eq. (4) since $\hat{\mu}_{1} \| \hat{\mu}_{2}$. Therefore only one polarization of the incident light can couple to the resonant modes of the dimer in the first place, barring any significant response for the orthogonal polarization.

\section{Discussion}

To discuss the optical activity properties of the PMMs, it is useful to determine their optical response with respect to their circular polarization components. The components of the circular-polarization transmission matrix $t$ are recovered from the linear-polarization coefficients $\tau_{i j}$ by the standard

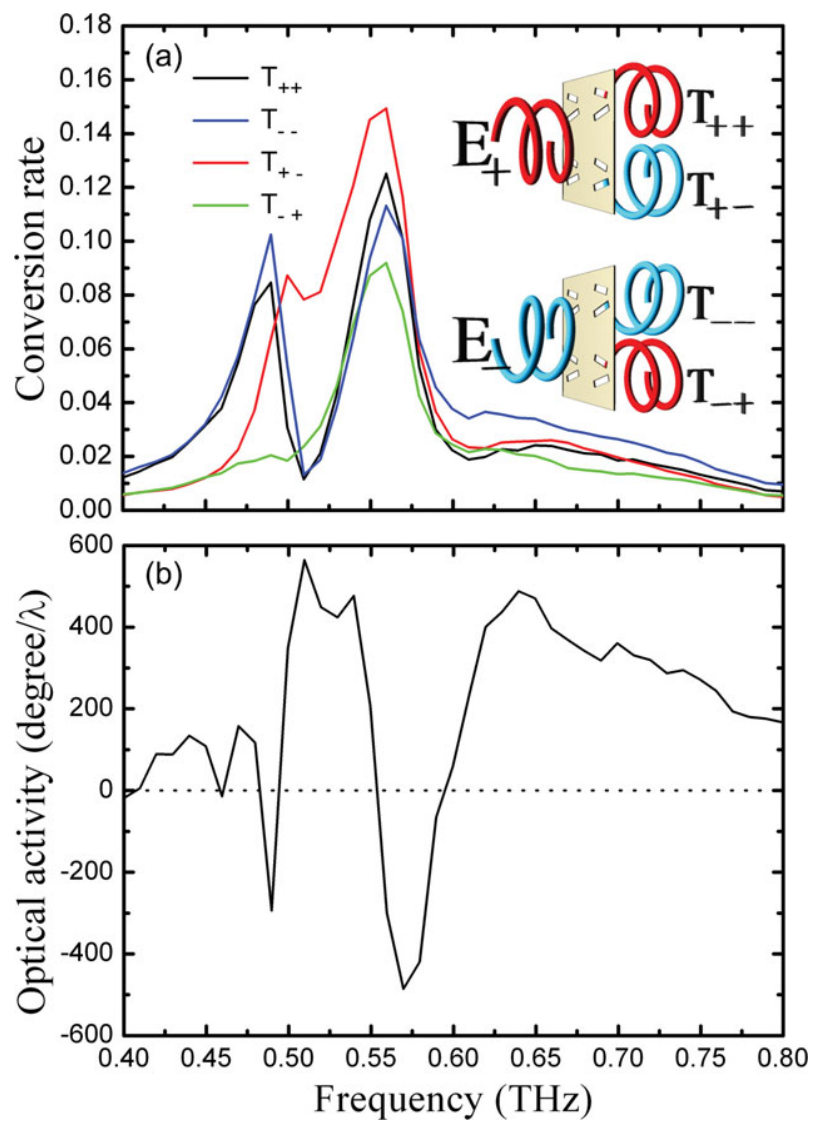

Figure 7 (a) Relative transmission $\left(\mathrm{T}_{++}, \mathrm{T}_{-}\right)$and circular polarization conversion $\left(T_{+-}, T_{-+}\right)$in terms of power for $\mathrm{V}$-shape slot planar metamaterial. (b) Optical activity for V-shape antirod slot metamaterial.

transformation

$$
\begin{aligned}
& \left(\begin{array}{l}
t_{++} t_{+-} \\
t_{-+} t_{--}
\end{array}\right) \\
& =\frac{1}{2}\left(\begin{array}{l}
\tau_{x x}+\tau_{y y}+i\left(\tau_{x y}-\tau_{y x}\right) \\
\tau_{x x}-\tau_{y y}+i\left(\tau_{x y}+\tau_{y x}\right) \\
\tau_{x x}+\tau_{y y}-i\left(\tau_{x y}+\tau_{y x}\right) \\
\left.\tau_{x y}-\tau_{y x}\right)
\end{array}\right) .
\end{aligned}
$$

The resulting power transmittances $\left(T_{++}=\right.$ $\left.\left|t_{++}\right|^{2}, T_{--}=\left|t_{--}\right|^{2}\right)$ and circular polarization conversion coefficients $\left(T_{+-}=\left|t_{+-}\right|^{2}, T_{-+}=\left|t_{-+}\right|^{2}\right)$ extracted from the measurements are presented in Fig. 7(a). We observe a pronounced anisotropic transmission behavior for the V-shaped Babinet PMM. Although the transmission of the right-handed polarization component $\left(T_{++}\right)$is almost equal to the left-handed polarization component $\left(T_{--}\right)$, the circular polarization conversion rates are significantly different. In the frequency range from 0.49 to $0.52 \mathrm{THz}$, the conversion difference between left-to-right $\left(T_{-+}\right)$and right-to-left $\left(T_{+-}\right)$circular polarizations is approximately $65 \%$, coinciding with the frequency band of high transmission. 
Since no noticeable difference is observed for $T_{++}$ and $T_{--}$we conclude that the $\mathrm{V}$-shape antirod planar structure exhibits no sizable circular dichroism within a broad frequency band. However, the PMM has pronounced elliptical dichroism, as discussed in section 4 . This results in significant optical activity, reaching a maximum of the polarization plane rotation rate of approximately $500 \% / \lambda$ at $0.53 \mathrm{THz}$ (Fig. 7(b)). Such huge optical activity is comparable with performance of more complex (dual layer) designs reported previously $[28,29]$.

The corresponding parallel Babinet PMM does not exhibit any optical activity. This shows that the asymmetric design of the $\mathrm{V}$-shaped slot dimers is crucial for the anisotropic transmission behavior.

\section{Conclusions}

In conclusion, we have developed a new process for fabricating high aspect ratio, freestanding metal films. We have fabricated and characterized a terahertz anisotropic Babinet planar metamaterial designed as a macroscopic, patterned $2 \mu$ m-thick Ni membrane suspended in air. We find very good agreement between the design and the fabricated dimensions, as evidenced by the excellent agreement between the numerical simulations and experimental measurements. Despite its relative simplicity, our V-shaped slot design shows optical activity comparable with two and three dimensional chiral materials, reaching a maximum of approximately $500 \% / \lambda$ at $0.53 \mathrm{THz}$, and a polarization conversion difference between left-to-right and right-to-left circular polarization of approximately $65 \%$ in the range between 0.49 and $0.52 \mathrm{THz}$. By optimizing the angle and distance between the rods even stronger circular polarization conversion may be achieved. The presented fabrication flow is a versatile platform for other designs that are sensitive to thick substrates and we believe that due to the simple design and flexibility of the PMM, it has a great potential to be integrated as a component in THz optical circuitry.

Acknowledgement. The authors acknowledge Dr. Andrew Strikwerda for fruitful discussions and text proof reading. Partial financial support from the Danish Research Council for Technology and Production Sciences through Project No. 09-070397-FTP is acknowledged. S. V. Z. acknowledges partial financial support from the People Programme (Marie Curie Actions) of the European Union's 7th Framework Programme FP7-PEOPLE-2011-IIF under REA grant agreement No. 302009 (Project HyPHONE).

Received: 1 March 2013, Revised: 8 May 2013, Accepted: 17 May 2013

Published online: 14 June 2013

Key words: metamaterial, terahertz, spectroscopy, optical activity.

\section{References}

[1] M. Tonouchi, Nature Photon. 2, 97-105 (2007).

[2] P. H. Siegel, IEEE Trans. Micowave. Theory Tech. 52, 24382447 (2004).

[3] P. U. Jepsen, D. G. Cooke, and M. Koch, Laser Photon. Rev. 5, 124-166 (2011).

[4] H.-T. Chen, J. F. O'Hara, A. K. Azad, and A. J. Taylor, Laser Photon. Rev. 5, 513-533 (2011).

[5] G. Kenanakis, R. Zhao, A. Stavrinidis, G. Konstantinidis, N. Katsarakis, M. Kafesaki, C. M. Soukoulis, and E. N. Economou, Opt. Mat. Express. 2, 1702-1712 (2012).

[6] R. Singh, E. Plum, W. Zhang, and N. I. Zheludev, Opt. Express. 18, 13425-13430 (2010).

[7] C. Soukoulis and M. Wegener, Nature Photon. 5, 523-530 (2011)

[8] J. Zhou, D. R. Chowdhurry, R. Zhao, A. K. Azad, H.-T. Chen, C. M. Soukoulis, A. J. Taylor, and J. F. O'Hara, Phys. Rev. B. 86, 035448 (2012).

[9] S. Zhang, Y.-S. Park, J. Li, X. Lu, W. Zhang, and X. Zhang, Phys, Rev. Lett. 102, 023901 (2009).

[10] X. Xiong, W.-H. Sun, Y.-J. Bao, M. Wang, R.-W. Peng, C. Sun, X. Lu, J. Shao, Z.-F. Li, and N.-B. Ming, Phys. Rev. B. 81, 075119 (2010).

[11] R. Singh, E. Plum, C. Menzel, C. Rockstuhl, A. K. Azad, R. A. Cheville, F. Lederer, W. Zhang, and N. I. Zheludev, Phys. Rev. B. 80, 153104 (2009).

[12] L. Wu, Z. Y. Yang, Y. Z. Cheng, Z. Q. Lu, P. Zhang, M. Zhao, R. Z. Gong, X. H. Yuan, Y. Zheng, and J. A. Duan, Opt. Express. 21, 5239-5246 (2013).

[13] K. Iwaszczuk, A. C. Strikwerda, K. Fan, X. Zhang, R. D. Averitt, and P. U. Jepsen, Opt. Express. 20, 635-643 (2012).

[14] H. Tao, A. C. Strikwerda, M. Liu, J. P. Mondia, E. Ekmekci, K. Fan, D. L. Kaplan, W. J. Padilla, X. Zhang, R. D. Averitt, and F. G. Omenetto, Appl. Phys. Lett. 97, 261909 (2010).

[15] H. Tao, J. J. Amsten, A. C. Strikwerda, K. Fan, D. L. Kaplan, X. Zhang, R. D. Averitt, and F. G. Omenetto, Adv. Mater. 22, 3527-3531 (2010).

[16] H. Tao, L. R. Chieffo, M. A. Brenckle, S. M. Siebert, M. Liu, A. C. Strikwerda, K. Fan, D. L. Kaplan, X. Zhang, R. D. Averitt, and F. G. Omenetto, Adv. Mater. 23, 3197-3201 (2011).

[17] S. V. Zhukovsky, C. Kremers, and D. N. Chigrin, Opt. Lett. 36, 2278-2280 (2011).

[18] C. Rockstuhl and F. Lederer, Phys. Rev. B. 76, 125426 (2007).

[19] F. Falcone, T. Lopetegi, M. A. G. Laso, J. D. Baena, J. Bonache, M. Beruete, R. Marqués, F. Martin, and M. Sorolla, Phys. Rev. Lett. 93, 197401 (2004).

[20] L. Zhang, T. Koschny, and C. M. Soukoulis, Phys. Rev. B. 87, 045101 (2013).

[21] F. J. Garcia-Vidal, L. Martin-Moreno, T. W. Ebbesen, and L. Kuipers, Rev. Mod. Phys. 82, 729-787 (2010).

[22] Y. M. Bahk, J.-W. Choi, J. Kyoung, H.-R. Park, K. J. Ahn, and D.-S. Kim, Opt. Express. 20, 25644-25653 (2012).

[23] D. Rakic, A. B. Djurisic, J. M. Elazar, and M. L. Majewski, Appl. Opt. 37, 5271-5283 (1998). 
[24] D. N. Chigrin, C. Kremers, and S. V. Zhukovsky, Appl. Phys. B. 105, 81-97 (2011).

[25] J.-H. Choe, J.-H. Kang, D.-S. Kim, and Q-H. Park, Opt. Express. 20, 6521-6526 (2012).

[26] G. N. Borzdov, J. Phys. A: Math. Gen. 34, 6249-6257 (2001).
[27] L. Barkovskii, G. Borzdov, and A. V. Lavrinenko, J. Phys. A: Math. Gen. 20, 1095-1106 (1987).

[28] S. Engelbrecht, M. Wunderlich, A. M. Shuvaev, and A. Pimenov, Appl. Phys. Lett. 97, 081116 (2010).

[29] Z. Li, K. B. Alici, E. Colak, and E. Ozbay, Appl. Phys. Lett. 98, 161907 (2011).

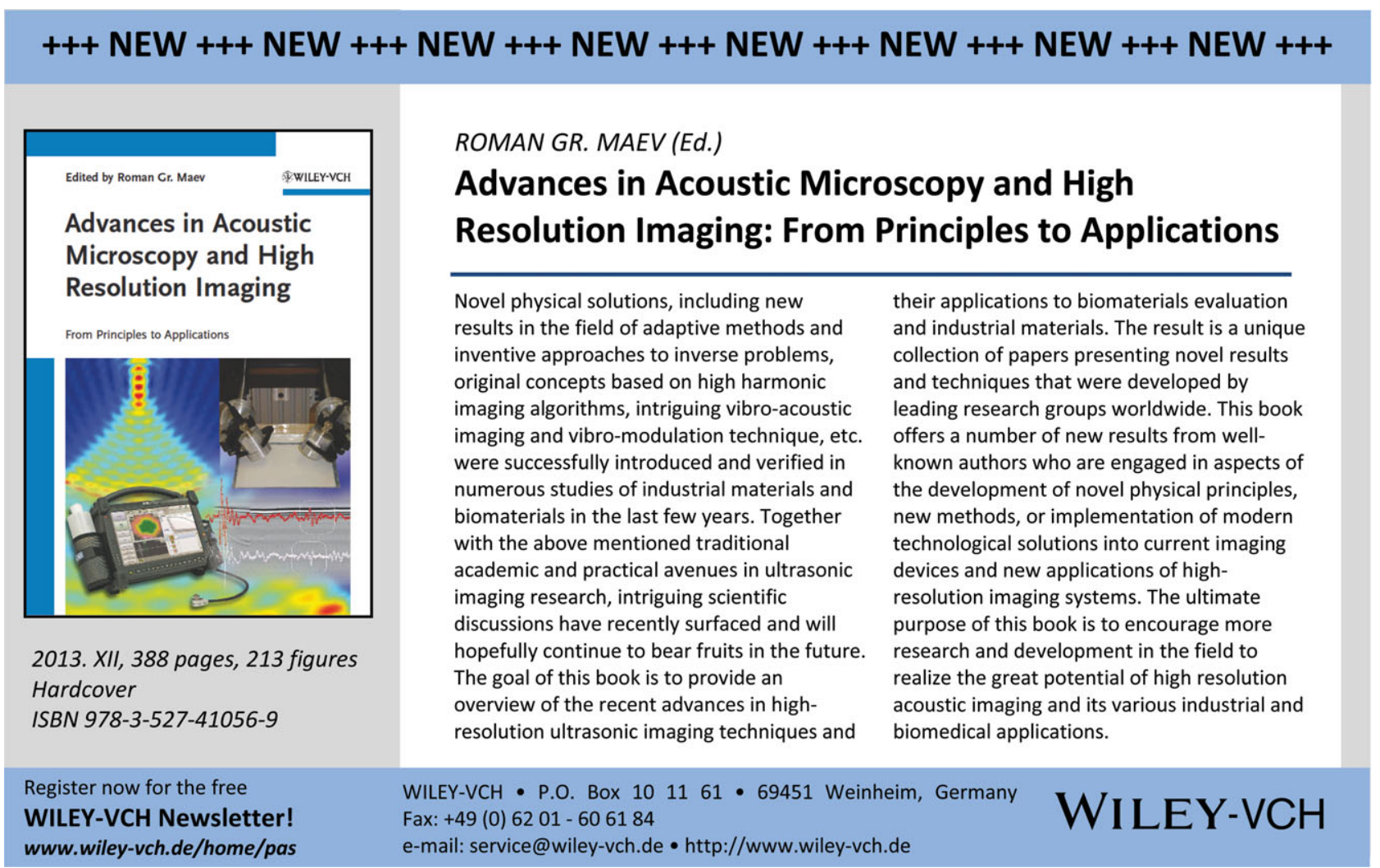

